$56^{\text {ème }}$ Congrès de la SFMBCB, 03006 (2011)

DOI: $10.1051 / \mathrm{sfmbcb} / 20115603006$

(C) Owned by the authors, published by EDP Sciences, 2011

\title{
Céphalée majorées par une avulsion dentaire : difficultés diagnostiques
}

\author{
Faby $\mathbf{N}^{1,2}$, Ogereau $\mathrm{F}^{2}$, Alno $\mathrm{N}^{2}$, Massot $\mathbf{M}^{2}$ \\ ${ }^{1}$ Service de Stomatologie, CHPC, Cherbourg, France \\ ${ }^{2}$ Service de Chirurgie buccale, CHU, Rennes, France \\ natacha.faby@wanadoo.fr
}

Les céphalées sont pour la plupart d'ordre fonctionnel et l'examen clinique avec une anamnèse précise suffit souvent à poser le diagnostic étiologique. Cependant, il arrive qu'elles constituent le symptôme d'une pathologie sous-jacente. En particulier, certaines formes surviennent brutalement et nécessitent une hospitalisation avec prise en charge rapide (Stefanizzi 2009).

MG, 72 ans, est adressé au Service d'urgences par son médecin traitant pour la prise en charge de céphalées apparues il y a un mois et s'accentuant malgré la prescription d'AINS. (acide niflumique). Ce patient souffre d'hypertension artérielle depuis vingt ans, traitée par une association d'énalapril/ercanidipine et fut opéré il y a quinze ans d'une hypertrophie bénigne de la prostate. Il est atteint par une arthrose cervicale et scapulaire responsable de douleurs depuis un an. Son médecin précise que sa fille est décédée de la rupture d'un anévrisme. Au cours des dix derniers jours, il a présenté un amaigrissement de quatre kilos ainsi qu'une asthénie mais pas de fièvre. Au bilan neurologique, on note une altération bilatérale de la fonction oculomotrice. Les épaules présentent une sensibilité à la mobilité sans diminution de l'amplitude fonctionnelle. Sur le plan cardiovasculaire, on évoque un possible souffle systolique ou un foyer mitral «à vérifier ». D'autre part, un syndrome inflammatoire est mis en évidence (augmentation du nombre des leucocytes et de la CRP). Le patient fait une relation entre l'aggravation de ses céphalées et l'avulsion d'une incisive mandibulaire réalisée quatre jours auparavant sous antibiothérapie (association amoxicilline/acide clavulanique); il est quasiment édenté. Les douleurs qu'il décrit sont continues, lancinantes, unilatérales droites, irradiant depuis la mandibule jusqu'au sommet du crâne, insomniantes, sans stimulation sensorielle ni zone gâchette. Elles sont soulagées pour de courtes durées par l'association paracétamol-dextropropoxyphène ainsi que par la compression manuelle exercée par le patient sur son cuir chevelu. Ces céphalées sont également provoquées par la palpation de la branche montante de la mandibule et de l'articulation temporo-mandibulaire droites ainsi que par la mastication. On observe une bonne cicatrisation muqueuse du site d'avulsion et l'orthopantogramme ne décèle pas de foyer infectieux d'origine dentaire. Une étiologie vasculaire est évoquée tandis que le premier examen ORL conclut à un SADAM provoqué par la perte de dimension verticale, la récente modification de l'occlusion et l'absence de prothèse mandibulaire. A partir de ces éléments, différentes hypothèses diagnostiques sont discutés : hypertension intra-crânienne, maladie de Horton, anévrisme, hématome sous-dural, abcès cérébral ou empyème post-chirurgical (Higelin 1999). La nette augmentation de la VS fait demander une seconde consultation en ORL, et la biopsie de l'artère temporale conclut à une artérite temporale ou maladie de Horton, affection dont la prévalence controversée est estimée à 1/1000 habitants (Ekbom 2006). Un angioscan aurait dû d'emblée être réalisé étant donné le contexte familial afin d'éliminer la possibilité d'un anévrisme. La conduite à tenir est ensuite décrite. Les difficultés diagnostiques des céphalées résident dans le fait qu'elles se situent au carrefour de nombreuses spécialités. L'examen clinique et surtout l'anamnèse doivent permettre d'orienter d'emblée les investigations et de réaliser une éventuelle prise en charge en urgence. 